\title{
Physics of humans, physics for society
}

To the Editor - Today, the massive use of information and communication technologies (ICT) has made it possible to attach a traceable set of data to almost any person. We argue that these data provide the opportunity to build a 'physics of society': describing a society composed of many interacting heterogeneous entities (people, businesses, institutions) - as a physical system. While important ethical implications have to be taken into account, the benefits in developing such physics of society would be tremendous. Indeed, it could help understanding, anticipating and forecasting future societal trends and human behavioural responses, and their associated uncertainty ${ }^{1}$; or address societal challenges in which globally networked risks play a role ${ }^{2,3}$. A case in point is modern epidemiology and its success in predicting the large-scale spreading of infectious diseases ${ }^{4}$.

The development of such a physics of society wouldn't be a bed of roses though. Humans are not molecules of an ideal gas. In the context of social dynamics, we don't understand yet whether 'Newtonian' laws exist for individuals. It is also unclear how many and which quantities would be needed for determining and anticipating a given macroscopic, in the sense of collective, observable. People have also the capacity to adapt, adding a degree of uncertainty or variability that cannot be neglected in many challenging problems, like the spreading of diseases. This means that the basic quantities we should work with must come from behavioural data.

Looking at behaviour, however, introduces another source of difficulty, as not all individuals are the same. As a matter of fact, the very notion of 'representative agent' - as a typical, perfectly rational and omniscient entity - has been criticized since its introduction in economics ${ }^{5}$. Actually, societal changes may be triggered by just a few key individuals - what is referred to as the law of the few ${ }^{3,7}$. This implies that, even if a suitable description of individual behaviour were available, scaling it up to groups of individuals wouldn't be straightforward, because the fluctuations with respect to the average can be large.

Indeed, this is what the data tell us: groups of individuals tend to be described by fattailed probability distributions, incomes are distributed according to Pareto's law ${ }^{8}$ and friendships in social networks follow power laws ${ }^{9}$. The quantities described by these fat tails have in some particular cases fluctuations so wide as to make it highly inaccurate to talk in terms of average behaviour. In other words, in the spirit of thermodynamics, we would ideally like to find quantities similar to pressure the integral of the particles' impulse - but, since individuals do not follow a well-behaved Maxwell-Boltzmann distribution function, this could simply be unachievable.

Yet, despite all of the above, we argue that physics can still play a pivotal role in the quest to find regularities in societal dynamics based on patterns of human interactions. Sociotechnical systems where human interactions are partly mediated by ICT exhibit emerging dynamics and are more often than not out of equilibrium. They also involve many temporal and spatial scales, are governed by nonlinear effects and can adapt to external and internal perturbations. We have roughly all the ingredients of a truly outof-equilibrium complex system, for the study of which physics provides methods and tools.

How should we proceed? The first step, in the very best tradition of physics, would be to make sense of the data. The most promising approaches to extract patterns from these data are methods developed in the context of artificial intelligence such as machinelearning techniques, which have been applied already in more traditional areas of physics.

But physics is not only gathering, mining and analysing data; it is also modelling of phenomena such as diffusion (of news, diseases, financial distress) or cluster formation (parties, trade zones). Indeed, while methods for data analysis can efficiently extract information from the data, they lack explanatory power, and do not necessarily increase our understanding of the world. As a second step, physics should thus produce first-principles approaches that are key to generating, for instance, 'what if' scenarios in modelling large-scale disease outbreaks.

The art of modelling, through definition, calibration and validation of models, is wellestablished in physics. It could then be used, in collaboration with the social sciences, for the development of mechanistic and theoretical models that could contribute to a better understanding of (technology-mediated) human interactions and societal dynamics. Their development would require going through the full research cycle, including testing theoretical predictions. Ultimately, the goal would be to find relevant quantities for describing societal and sociotechnical dynamics at a microscopic and macroscopic level and to connect them, similar to the way thermodynamics works, going from the smallest scale - the individual - to the largest - society.

In summary, the vision and mindset of physics are not only useful but we would say necessary to address social problems. An example is data-driven stochastic microsimulation of the Zika virus epidemic $^{10}$, which helps our understanding of a global and socially relevant dynamics, and at the same time contributes to the more traditional problem of characterizing diffusion in disordered media.

However, the pursuit of a physics for society would bear fruit only if physicists were ready to leave their traditional comfort zone and establish unconventional collaborations with researchers in other disciplines such as computer and social scientists, and economists, in addition to mathematicians. We believe that such vision and agenda can only be realized with the support of international organizations like the European Commission, which triggered the development of the field with its Future and Emerging Technology (FET) initiatives. These are aimed at developing consortia activity across Europe rather than individual research. Continuing this approach would provide the necessary support to the multi- and trans-disciplinary initiatives that can enrich and exploit existing methods, paving the way for a statistical physics of the future that will contribute to the development of a whole new theoretical and computational body of knowledge of human interactions and societal dynamics.

By defining values and objectives through the involvement of stakeholders and citizens, we believe that this body of knowledge could allow to successfully co-design a sustainable and fair future society.

\section{Guido Caldarelli, 1,2, , Sarah Wolf ${ }^{4}$ and Yamir Moreno ${ }^{5,6}$ \\ ${ }^{1}$ Scuola IMT, Lucca, Italy. ${ }^{2}$ Institute of Complex Systems, CNR, Rome, Italy. ${ }^{3}$ ECLT, Venice, Italy. ${ }^{4}$ Global Climate Forum, Berlin, Germany. ${ }^{5}$ Institute for Biocomputation and Physics of Complex Systems, University of Zaragoza, Zaragoza, Spain. ${ }^{6}$ ISI Foundation, Turin, Italy. ${ }^{*}$ e-mail: guido.caldarelli@gmail.com}

Published online: 4 September 2018 https://doi.org/10.1038/s41567-018-0266-X

\footnotetext{
References

1. Nature 453, 698 (2008).

2. Battiston, S. et al. Science 351, 818-819 (2016).

3. Helbing, D. Nature 497, 51-59 (2013).

4. Vespignani, A. Nat. Phys. 8, 32-39 (2012).

5. Kirman, P. A. J. Econ. Perspect. 6, 117-136 (1992).

6. Gladwell, M. The Tipping Point: How Little Things Can Make a Big Difference (Little, Brown, Boston, MA, 2000).

7. Galeotti, A. \& Goyal, S. Am. Econ. Rev. 100, 1468-1492 (2010).

8. Pareto, V. Cours d'Economie Politique (Droz, Geneva, 1896).

9. Lazer, D. et al. Science 323, 721-723 (2009).

10. Zhang, A. et al. Proc. Natl Acad. Sci. USA 114, E4334-E4343 (2017)
} 